\title{
Unvollständige Daten in randomisierten dermatologischen Studien: Auswirkungen und statistische Methoden
}

\section{Schlüsselwörter}

Inverse Wahrscheinlichkeitsgewichtung · Fortschreibung des letzten gemessenen Wertes (last observation carried forward) Fehlende Daten · Multiple Imputation · Non-ResponderImputation

\footnotetext{
Zusammenfassung

Randomisierte klinische Studien können den höchsten Evidenzgrad für die Wirksamkeit einer therapeutischen Maßnahme liefern. Wenn einzelne Teilnehmer vorzeitig aus einer Studie herausfallen, ist es häufig nicht möglich, die gewünschten Verlaufsparameter zu ermitteln; das führt zu unvollständigen Daten. Die vorliegende Arbeit gibt einen Überblick über verschiedene Mechanismen, die zu unvollständigen Daten führen können. Außerdem werden die Auswirkungen dieser Mechanismen diskutiert und Strategien für
} den Umgang mit unvollständigen Daten vorgestellt. Die genannten Aspekte werden im Kontext klinischer dermatologischer Studien besprochen. Weiterhin werden praktische Empfehlungen für die Planung von Studien und die Interpretation von Ergebnissen aus Studien mit möglicherweise unvollständigen Daten gegeben.

C 2014 S. Karger GmbH, Freiburg

\footnotetext{
Originalartikel

Incomplete Data in Randomized Dermatology Trials: Consequences and Statistical Methodology

Michael A. Mclsaac ${ }^{\mathrm{a}}$ Richard J. Cook ${ }^{\mathrm{a}}$ Melanie Poulin-Costellob

aDepartment of Statistics and Actuarial Science, University of Waterloo, Waterloo, ON, bAmgen, Mississauga, ON, Canada
} 


\section{Warum ist der Artikel relevant für den deutschen Praxis- und Klinikalltag?}

Patientendaten, die in klinischen Studien erhoben werden, sind eine wertvolle Ressource. Sie sind so genau und vollständig wie möglich auszuwerten.

Fehlende Daten (missing values) und die daraus resultierende Nichtberücksichtigung von Patienten in der Auswertung stellen auf der einen Seite ein ethisches Problem dar (Belastung des Patienten durch die Studienteilnahme). Auf der anderen Seite können fehlende Daten verhindern, dass das Studienziel erreicht wird.

In dermatologischen Studien werden häufig Auswertungsverfahren und Strategien für den Ersatz fehlender Daten verwendet, die das Studienergebnis - unkritisch angewendet - ungewollt verzerren können. Modernere Strategien zum Ersatz von Daten kommen bisher nur selten zum Einsatz.

\section{Welche Inhalte/Behandlungsoptionen sind neu?}

Der hier kommentierte Artikel erläutert die Klassifikation von fehlenden Daten (missing completely at random (MCAR), missing at random (MAR), missing not at random (MNAR)) sowie die Relevanz dieser Einstufungen. Die gängigen Verfahren für die Auswertung (complete-case analysis (CCA)) und für den Ersatz fehlender Werte (Imputationsstrategien wie last observation carried forward (LOCF) oder non-responder imputation (NRI)) werden im Hinblick auf diese Klassifikation beurteilt und ihre Einsatzmöglichkeiten aufgezeigt. Zudem macht der Beitrag die Konsequenzen des falschen Einsatzes dieser Verfahren deutlich.

Diese Betrachtungsweise ist ein Novum für viele dermatologische Forschungsvorhaben. Um den Einfluss fehlender Werte auf dermatologische Parameter zu verringern, wird die Betrachtung von Zeitverläufen anstelle von Zeitpunkten empfohlen.

\section{Welche Aussagen sind kritisch zu bewerten?}

Die vielfach angesprochene Spezifikation von Modellen für den Ersatz fehlender Werte ist zwangsläufig sehr allgemein gehalten. Hier gilt es, jeweils einen Konsens für die zu messenden
Parameter, die als Endpunkte eingesetzt werden, zu finden. Dazu ist Grundlagenarbeit in Abhängigkeit von den Parametern zu leisten. Die Modelle müssen allgemein akzeptiert und aufeinander abgestimmt werden.

Hinweise auf bereits vorliegende Modelle für bestimmte Parameter wären hilfreich, um sich ein Bild vom Aussehen eines solchen Modells machen zu können.

\section{Welche Perspektive eröffnen die Ergebnisse für die Behandlungspraxis?}

Zur Planung einer klinischen Studie gehört die Beantwortung folgender Fragen:

- Welche fehlenden Daten sind für die Auswertung relevant?

- Welchem Mechanismus folgen diese fehlenden Daten?

- Welche Imputationsstrategie ist für diese Daten angemessen?

- Gibt es ein akzeptiertes Modell, dem diese Daten folgen?

- Welche Konsequenzen werden aus einer Sensitivitätsanalyse gezogen, die die Studienergebnisse ohne und mit imputierten Daten (gegebenenfalls nach verschiedenen Verfahren) vergleicht?

Durch die Klärung dieser Fragen zum Umgang mit fehlenden Daten im Vorfeld der klinischen Durchführung einer Studie lassen sich Verzerrungen von Ergebnissen vermeiden oder zumindest minimieren.

Der Artikel stellt einen wertvollen Impuls für eine weitere Qualitätssteigerung von Studien dar. Er ermutigt dazu, sich bereits im Vorfeld bewusster mit fehlenden Daten und deren individuelleren Auswertung auseinanderzusetzen.

Die dazu notwendigen Modelle müssen in Abhängigkeit von der Grundkrankheit und den jeweils relevanten Parametern entwickelt und diskutiert werden. Dadurch kann der Ersatz fehlender Daten und die Auswertung von Studien mit solchen fehlenden Daten in einer nachvollziehbareren Form erfolgen.

Kontaktadresse: Dr. phil. Ina Zschocke, SClderm GmbH, Drehbahn 1-3, 20354 Hamburg, Deutschland 